\title{
Los ensayos no destructivos (END) y su aplicación en la industria
}

Non-destructiver testing (NDT) and its application in the industry

Recibido: diciembre 05 de 2017 | Revisado: febrero 15 de 2018 | Aceptado: abril 13 de 2018

Jorge Luis Calderón Cáceres ${ }^{\mathrm{T}}$
Gian Carlo Scarpati Gálvez ${ }^{2}$

\section{Resumen}

Es de vital importancia para los ingenieros que se desempeñan en la industria, el conocimiento y destreza en la aplicación de los ensayos no destructivos, teniendo en cuenta que constituyen una herramienta de primera línea en la detección de fallas o defectos.

Palabras clave: Ensayos no destructivos, industria, detección

\begin{abstract}
It is of vital importance for the engineers who work in the industry, the knowledge and skill level in the application of nondestructive tests, considering that they constitute a primary tool in the detection of faults or defects.
\end{abstract}

Key words: Non-destructive tests, industry, detection

1 Universidad de San Martín de Porres, Perú jcalderonc@usmp.pe

2 Universidad de San Martín de Porres, Perú gscarpatig@usmp.pe

https://doi.org/ 10.24265/campus.2018.v23n25.05

| Campus | Lima, Perú | V. XXIII | N. 25 | PP. 59-66| ENERO-JUNIO | 20 I8 | ISSN I8I12-6049 


\section{Introducción}

La historia de los ensayos no destructivos puede considerarse que se remonta al siglo XIX con las investigaciones del británico William Crookes, quien desarrolló experimentos con tubos al vacío y electrodos generando corrientes de alto voltaje, lo que dio paso al descubrimiento de los rayos X en 1895 por el físico alemán Wilhelm Conrad Röntgen que permitió captar estructuras óseas. Así se inició a lo que en medicina se denomina procedimientos no invasivos; y posteriormente, aprovechar este descubrimiento para realizar el ensayo no destructivo, denominado radiografía industrial.

Es de vital importancia comprender que los ensayos no destructivos solo pueden ser practicados por personal calificado y certificado, considerando que una persona certificada ha demostrado su competencia para realizar END de acuerdo con cuál de los tres niveles a los que dicho operador haya alcanzado.

Según la ASNT, "Se denomina ensayo no destructivo a cualquier tipo de prueba practicada a un material que no altere de forma permanente sus propiedades físicas, químicas, mecánicas o dimensionales". Los ensayos no destructivos han evolucionado como un elemento esencial en los diseños de ingeniería modernos, procesos y tecnologías de producción, mantenimiento y diagnóstico. Por lo tanto, la capacidad de detección y la fiabilidad de los procedimientos no destructivos son de vital importancia para el uso final del elemento inspeccionado, y responden a los desafíos y necesidades de la industria manufacturera en general.
En virtud de la importancia de la utilización de esta herramienta, y con el fin de normar su actividad, es que en la mayoría de países -industrializados existen sociedades de ensayos no destructivos, destacando a nivel mundial la ASNT (American Society For Nondestructive Testing), la CHSNDT (Chinese Society For Nondestructive Testing), y la EFNDT (European Federation For Nondestructive Testing), que agrupa a las sociedades europeas respectivas de los diferentes países. Entre los principales ensayos no destructivos se encuentran: corrientes inducidas o corrientes Eddy, inspección por líquidos penetrantes (tintes) y, la radiografía industrial.

El presente artículo describe tres de los principales ensayos no destructivos, a saber: por inducción electromagnética, líquidos penetrantes y por rayos $\mathrm{X}$.

\section{Ensayos no destructivos por inducción electromagnética}

El método no destructivo de ensayo por corrientes inducidas, también llamadas corrientes de Faucault o Eddy currents (corrientes de torbellino) se basa en el principio de la inducción electromagnética (E.D.E.N. 2009). Debido a la versatilidad de este tipo de ensayo es posible resolver problemas relacionados con:

1. Medición de parámetros físicos (conductividad, permeabilidad, tamaño de grano, dureza).

2. Detección de discontinuidades, entre ellas: corrosiones, inclusiones, segregaciones, grietas. (Figura 1)

3. Separación de metales mezclados (composición química, medida de espesores de recubrimiento). 


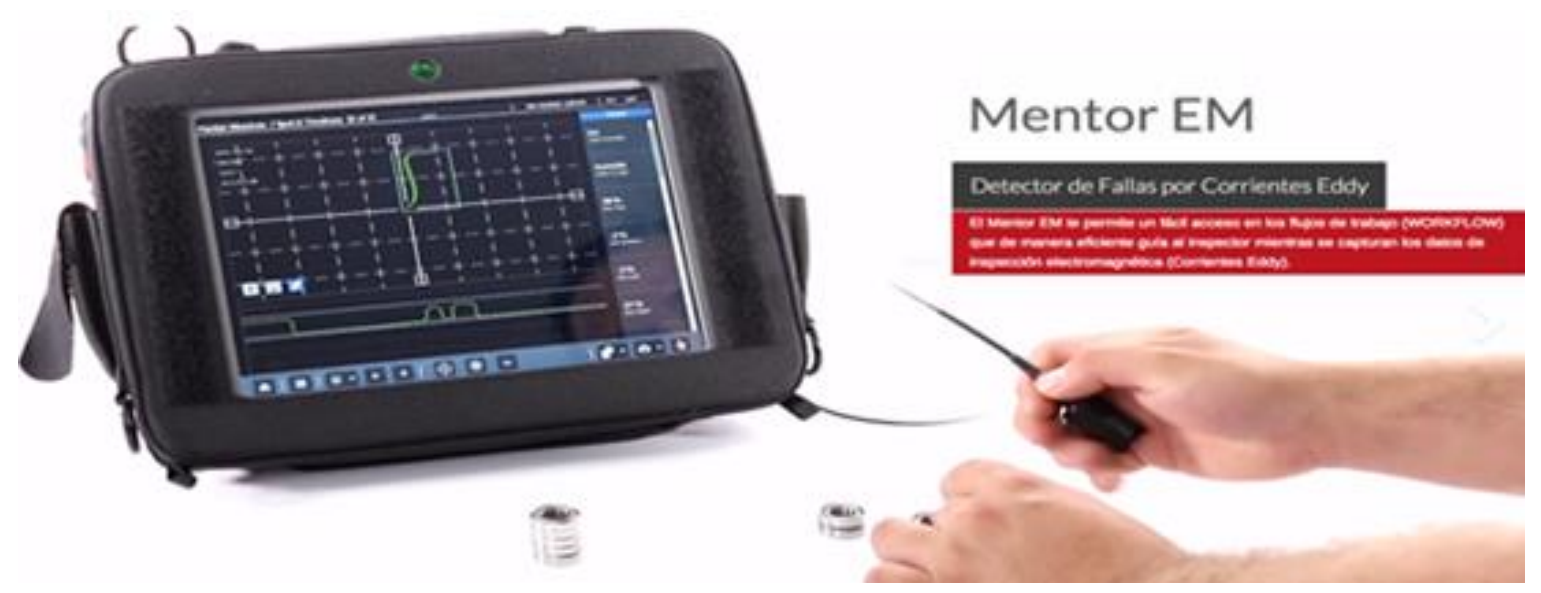

Figura 1. Detector de Fallas por Corrientes Eddy.

\section{Ensayos no destructivos por líquidos penetrantes}

Este tipo de ensayo se utilizó en una planta química llamada Alcalis Peruanos de la Empresa Sociedad Paramonga Limitada, hoy QUIMPA S.A.. El ensayo aplicado en esta planta de cloro - soda, específicamente, en un tanque de cloro, debido a una fuga no identificada, siendo utilizada para detectar en posibles porosidades, grietas, juntas en la superficie cilíndrica. El equipo utilizado para realizar

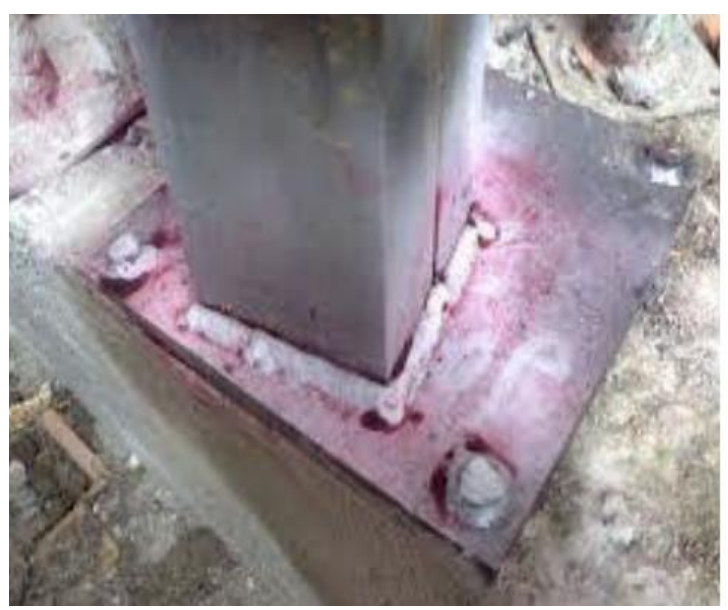

Figura 2. Ensayo por líquidos penetrantes

Siguiendo el procedimiento, se aplica el tinte color rojo intenso por rociado, por inmersión y también de aplicación utilizando para ello una brocha o por ro- el ensayo en campo estuvo compuesto por solventes, limpiador, un líquido denominado tinte y una suspensión de polvo en líquido utilizado como revelador.

El ensayo empezó con la limpieza de la superficie a inspeccionar hasta lograr un área libre de pintura, salpicaduras de soldadura, pintura y aceites, evitando así la contaminación que podría impedir el ingreso del tinte en las soldaduras discontinuas (Figura 2).

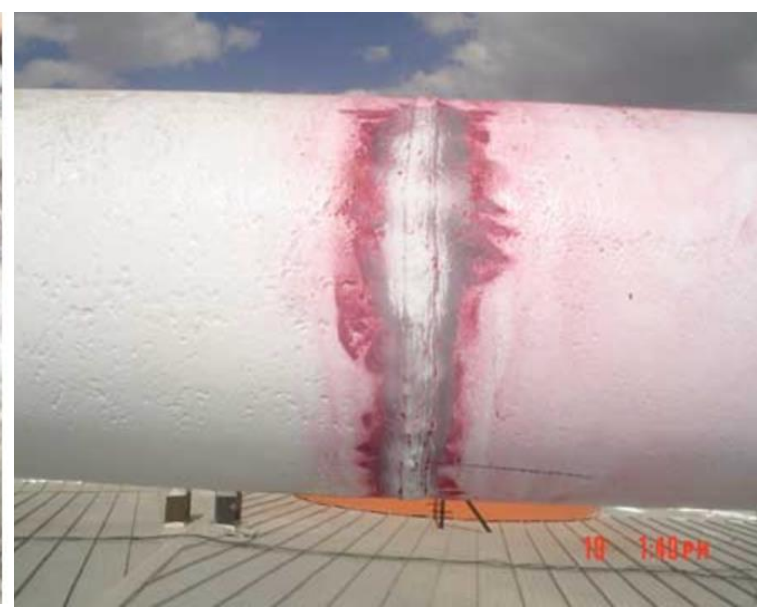

ciado, se espera un tiempo prudente y se limpia con agua. Acto seguido, se aplica una capa fina del revelador de color blanco en la superficie tratada. Esta suspen- 
sión provoca la exudación del tinte hacia la superficie, lo cual nos indica en la discontinuidad.

Con este ensayo es posible ubicar algunas grietas, e información sobre su naturaleza y dimensiones y efectuar las correcciones del caso. Con base en las dimensiones, las indicaciones pueden ser clasificadas de acuerdo con lo especificado en la norma ASTM E 433, Standard Reference Photographs for Liquid Penetrant Inspection (Fotografías de Referencia Estandarizadas para la Inspección por Tintes Penetrantes). Sobre la base de esta norma, las indicaciones pueden ser clasificadas en los tipos I y II. Las dimensiones en una indicación tipo I, son iguales $o$, en caso de ser diferentes, una dimensión nunca es mayor a tres veces la otra. Por el contrario, en las indicaciones tipo II, una dimensión es al menos tres veces mayor que la otra. Por consiguiente, las indicaciones tipo I tienen forma de poro, mientras que las indicaciones tipo II tienen el aspecto de una grieta. Los tipos mencionados pueden ser clasificados en las clases A, B, C y D, dependiendo de la distribución de la indicación.

La clase de categoría A se refiere a la presencia de una sola indicación; la clase B identifica a múltiples indicaciones no alineadas, la clase $\mathrm{C}$ se refiere a múltiples indicaciones alineadas, es decir, organizadas en una línea recta o curva; la clase $\mathrm{D}$ identifica a las indicaciones ubicadas en la intersección de dos superficies como, por ejemplo, aquellas asociadas a roscas de tornillos, agujeros y esquinas. Este ensayo por tintes penetrantes tam- bién puede realizarse con un trazador fluorescente, el cual requiere de una lámpara negra o de rayos ultravioleta para la observación de las indicaciones. El procedimiento de limpieza y aplicación de tinte y solvente, en este caso, es igual que en el caso previo.

\section{Ensayos no destructivos utilizando Ra- yos $\mathrm{X}$}

Técnicamente, estas pruebas, son conocidas como radiografías industriales. Las utilizamos para detectar discontinuidades internas, tales como grietas porosidades, faltas de fusión en uniones con soldadura, piezas de fundición y piezas forjadas.

Es un tipo de prueba que se aplica a un material de forma que no altere, permanentemente, sus propiedades físicas, químicas, mecánicas, etc.; es decir, sin aplicar a este daño alguno. Básicamente, los rayos $\mathrm{X}$ son radiaciones electromagnéticas invisibles, que tienen la capacidad de atravesar cuerpos opacos, con una longitud de onda de $10^{-8} \mathrm{~m}(10 \mathrm{~nm})$ a $10^{-13}$ $\mathrm{m}\left(10^{-4} \mathrm{~nm}\right)$ (Serway, 1995 , p. 658).

En un sistema de refrigeración industrial Borsig de la Empresa Liofilizadora del Pacifico, el cual utiliza el amoniaco como refrigerante, encontramos un condensador, el cual enfría el amoniaco. Este condensador no viene a ser, sino un intercambiador, el cual presentaba fuga en su placa principal, produciendo una mezcla de agua con amoniaco (Figura 3), la cual presentaba fuga entre algunos tubos y la placa de acero. 


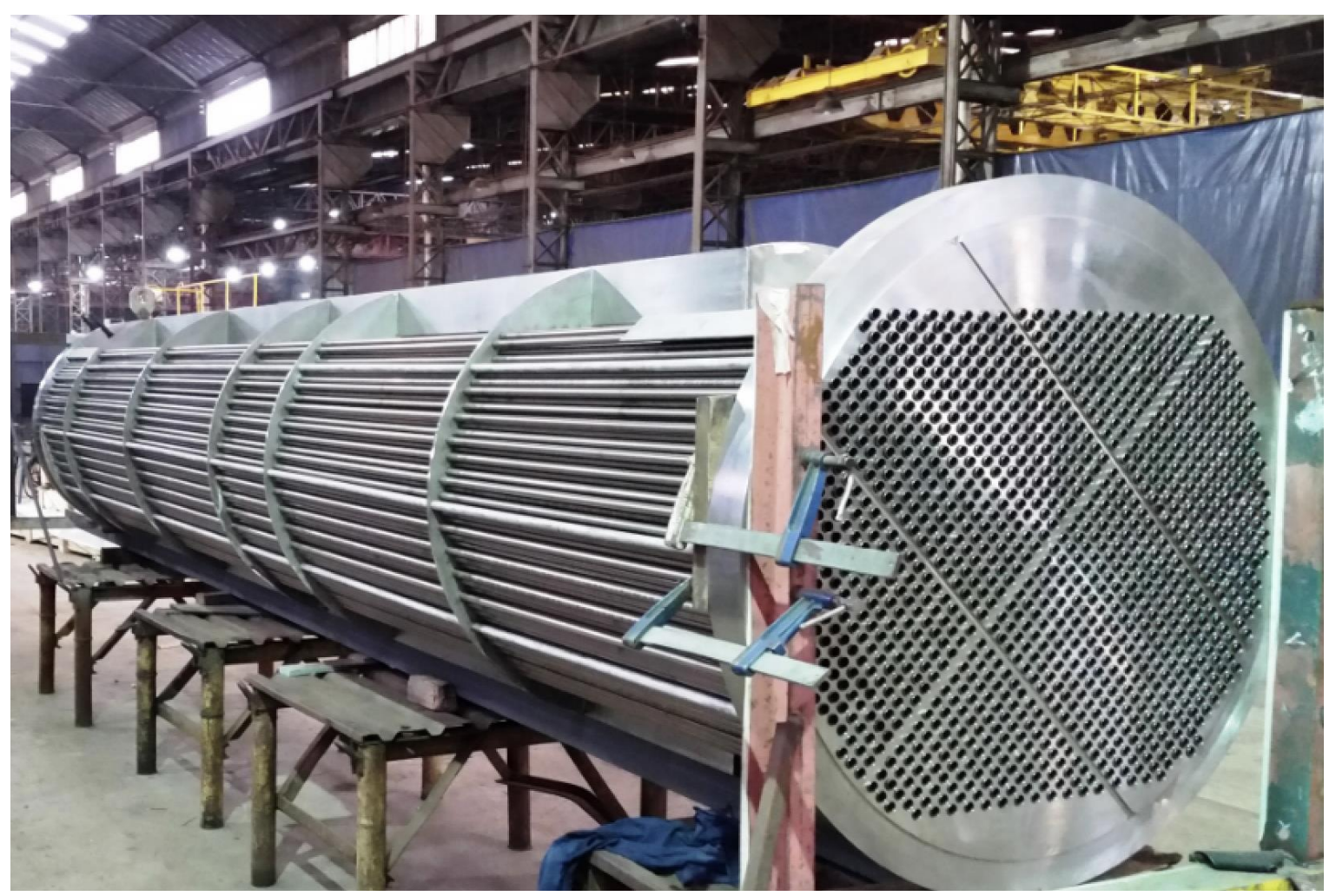

Figura 3. Placa metálica de un intercambiador de gran capacidad, antes de tomar las Placas de Rayos X

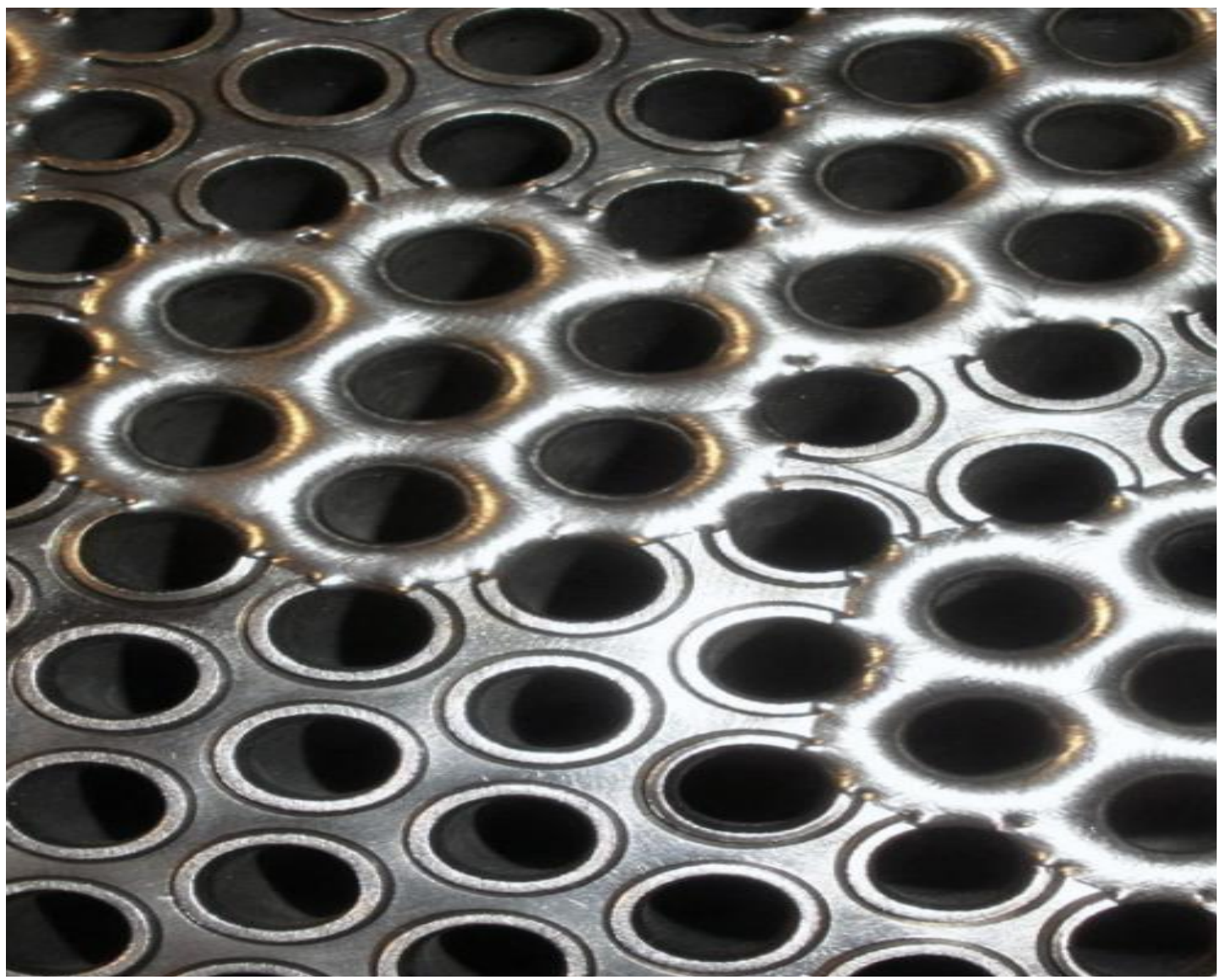

Figura 4. Tubos expandidos en la placa con problemas de sellado 
Por lo tanto, se puede afirmar que se ahorró tiempo, miles de dólares y se pudo cumplir con los pedidos de productos liofilizados, atendiendo a los pedidos del exterior, que incluye a Japón, Alemania, China y los Estados Unidos de Norteamérica.

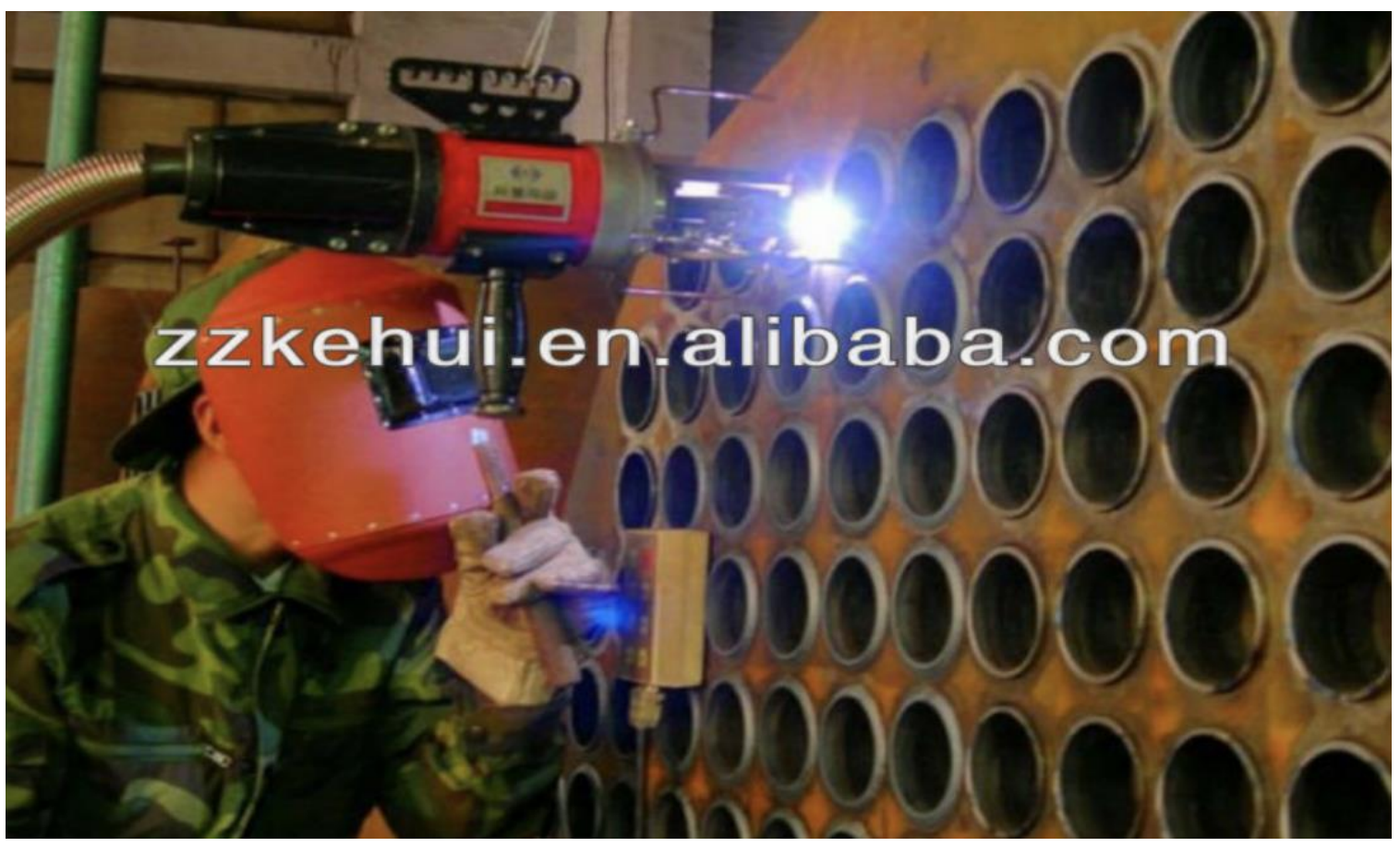

Figura 5. Máquina de soldadura automática

Siendo un excelente medio de registro de inspección, su uso se extiende a diversos materiales. Mediante este método se obtiene una imagen visual del interior del material y un registro permanente de la inspección, lo que permite descubre los errores de soldadura, fabricación y ayuda a establecer las acciones correctivas correspondientes. Para su aplicación, se encuentran disponibles para la industria equipos fijos y equipos portátiles, a elegirse según las características de la maquinaria objeto de análisis. En su empleo, como en todas las buenas prácticas industriales, se deben seguir estrictas medidas de seguridad (cabina de plomo, trajes, etc.). También se requiere de instalaciones especiales como son: el área de exposición, equipo de seguridad y un cuarto oscuro para el proceso de revelado.

\section{Conclusiones}

1. Se concluye que los ensayos no destructivos son una herramienta de inspección efectiva y eficiente, que permiten diagnosticar el estado de equipos sin deteriorarlos, pudiendo ser aplicados durante las diferentes etapas fabricación, montaje y en operación de los equipos industriales.

2. Las diferentes técnicas que envuelven a los ensayos no destructivos, aplicadas conveniente y oportunamente, previenen fallas que protegen la vida humana, la contaminación del medio ambiente y el ahorro en la conservación de los activos.

3. En el Perú, principalmente, las industrias relacionadas con la minería, hidrocarburos, pesquería, alimentos y la 
actividad manufacturera en general, se benefician directamente mediante la aplicación de estas tecnologías; Considerando que el presente año, produce después de cuatro años con cifras en rojo, la industria peruana podría acelerar su proceso de recuperación y crecería 3,7\% al término del 2018, siendo la pesca industrial y los metales primarios las principales actividades que promoverán el crecimiento de la manufactura primaria.

\section{Referencias}

E.D.E.N., (2009). Corrientes inducidas: nivel II. Recuperado de https:// ebookcentral.proquest.com

Burte, H; Chimenti, E. (1983). The Middle Ground of NDE R\&D Spectrum, Research of Progress in Quantitative Nondestructive Evaluation. New York: Plenum Press.

Lincoln, J. (1998). Role of Non Destructive Inspections in Airworthiness Assurance.

RTO AVT Workshop on Airframe Inspection Reliability under Field / Depot Conditions. Brussels Belgium.
Ludwin, F. (1972). Mantenimiento de las máquinas herramientas. Barcelona: Editorial Blume.

Roldán, J. (1997). Manual de mantenimiento de instalaciones. Madrid: Editorial Paraninfo.

Serway, R. \& Jewett, J. (2008). Física Tomo II. Séptima edición. México: Cengage Learning Editores S.A.

Tipler, P. (2000). Física Tomo II. Cuarta Edición. España: Reverté, S.A 